\title{
Experiencias y Logros sobre Mejoramiento Convencional y Selección Participativa de Cultivares de Papa en Bolivia
}

\author{
Julio L. Gabriel, Enrique Carrasco, Willman García, \\ Hermeregildo Equise, Oscar Navia, Rudy Torrez, Noel Ortuño, \\ Javier Franco, Graham Thiele, Nelson Estrada ${ }^{1}$
}

\begin{abstract}
Resumen
Desde 1989, PROINPA en Bolivia ha generado y obtenido por selección en el Banco de Germoplasma de papa y por cruzas ínter e intraespecífica, retrocruzamiento, selección recurrente, y selección participativa, nuevos cultivares más productivos y resistentes a los factores bióticos como el tizón Phytophthora infestans, el nematodo Nacobbus aberrans y la verruga Synchytrium endobioticum y tolerantes a factores abióticos como las heladas y sequía. Estos nuevos cultivares han sido seleccionados en Cochabamba, Chuquisaca, Potosí, Tarija, La Paz y Santa Cruz, por lo que varios de ellos tienen una amplia adaptación, buenas características agronómicas, altos rendimientos y calidad culinaria aceptable para el mercado. Se ha observado durante varios años que en la selección participativa de variedades de papa, en floración los criterios de selección fueron el vigor de la planta, la sanidad, y la resistencia a enfermedades. En la cosecha los criterios de selección fueron el rendimiento, el tamaño de tubérculos, la semejanza con otras variedades conocidas, la apariencia y color de la piel y la profundidad de ojos. En la evaluación culinaria (al mes de la cosecha) el agricultor tiende a comparar con su variedad local.
\end{abstract}

En diez años de intenso trabajo se han obtenido mediante mejoramiento genético convencional y selección participativa seis cultivares potenciales resistentes al tizón (India, Jaspe, Perla, Robusta, Chota Ñawi y Cordillera), dos resistentes al nematodo-rosario Nacobbus aberrans (Jaspe, Gendarme), tres resistentes a la verruga (Puka Uya, Amanecer, Pollerita), dos cultivares tolerantes a heladas (Illimani, Totoreña), y dos tolerantes a

1 Fundación PROINPA, Casilla 4285, Cochabamba, Bolivia.
Correo electrónico: jgabriel@proinpa.org. 
sequía (Potosina, Pampeña). Algunos de estos cultivares poseen resistencias combinadas para dos o tres factores abióticos y/o bióticos adversos.

Palabras claves adicionales: Híbridos ínter e intraespecíficos, tizón, nematodo, verruga helada, sequía, abiótico, biótico, selección participativa.

\section{Experiences and Outcomes on Conventional Plant Breeding and Participatory Potato Varieties Selection in Bolivia}

\section{Summary}

Since 1989, PROINPA in Bolivia has generated and obtained new cultivars through inter and intraspecific crosses, backcrossing, recurrent selection and participatory selection of varieties. These cultivars are more productive and resistant to abiotic and biotic factors like late blight (Phytophthora infestans), nematode (Nacobbus aberrans) and wart (Synchytrium endobioticum), and tolerant to abiotic factors as frost and drought. These cultivars were selected in Cochabamba, Chuquisaca, Potosí, Tarija, La Paz and Santa Cruz, some of them have wide adaptation, good agronomic characteristics, high yields and a good market quality. It has been observed during several years that in the participatory variety selection of potato at the flowering stage, the approaches of the farmers were the vigor of plant, sanirary condition, and resistance to disease. At harvest, the selection factors were yield, size of tubers, similarity to other well-known varieties, the appearance and color of the skin and the depth of eyes. In the culinary evaluation (one month after harvest) the farmer tends to compare with his local variety.

During ten years of study, we have selected, by conventional plant breeding and participatory varieties selection procedures, 6 potential cultivars resistant to late blight (India, Jaspe, Perla, Robusta, Chota Ñawi, and Cordillera), 2 resistant to Nacobbus aberrans (Jaspe, Gendarme), 3 resistant to wart (Puka Uya, Amanecer, Pollerita), 2 tolerant to frost (Illimani, Totoreña) and 2 tolerant to drought (Potosina Pampeña). Some of these cultivars have combined resistance against 2 or 3 abiotic and biotic factors. 
Additional Índex words: ínter and intraspecific, late blight, nematode, ward, frost, drought, abiotic, biotic, participatory selection.

\section{Introducción}

La papa es uno de los cultivos agrícolas más importantes en Bolivia y constituye un alimento básico de la dieta familiar. A él se dedican unas 400,000 familias, lo que equivale al $50 \%$ de las unidades agrícolas cultivadas del país. El consumo es de $98 \%$ en forma de papa fresca, sin procesar.

Sin embargo, la papa es vulnerable al ataque de un gran número de enfermedades y plagas. Hooker (1981) menciona 25 virus, 38 hongos, 6 bacterias, 2 micoplasmas y un viroide; además de 68 especies de nematodos, y 128 insectos-plaga, totalizando 266 patógenos y plagas. También diezman la papa factores abióticos como las heladas y la sequía.

En general las enfermedades y plagas que atacan a la papa, constituyen los principales factores limitantes para la obtención de rendimientos más altos. En Bolivia se considera a los virus como los principales factores de degeneración de la semilla, además la verruga (Synchytrium endobioticum) y nematodos (Nacobbus aberrans, Globodera spp.) como los principales factores negativos que limitan las áreas disponibles en el país para la producción de semilla de mejor calidad, que al momento es deficitaria. También es importante la marchitez bacteriana (Ralstonia solanacearum) de reciente introducción, la cual está actualmente anulando zonas productoras de semilla de gran potencial para el futuro de la producción de papa en Bolivia (PROINPA, 1996).

El tizón (Phytophthora infestans), de amplia distribución en las zonas paperas del país, causa actualmente pérdidas de hasta $100 \%$ en las cosechas de los agricultores y obliga a los mismos a realizar aplicaciones frecuentes de fungicidas que van en detrimento del ambiente y de la salud del agricultor y su familia (Estrada et al., 1994 a, 1994b, Gabriel et al., 1996a, PROINPA, 1996). 


\section{Etapas de la evolución de la papa}

Las primeras papas domesticadas pertenecieron a Solanum stenotumum, la cual se derivó de S. lepthophyes. A su vez S. stenotomum, se cruzó posiblemente con S. sparsipilum, que es silvestre diploide, para originar $S$. andigena precursor de la papa tetraploide actual (Estrada et al., 1994a; Hawkes, 1988).

\section{Potencial genético de las especies de papa}

La Escuela Vavilov en Leningrado de manera visionaria envió en 1920 a los doctores S.M. Bukasov y Juzepczuk para realizar las primeras colecciones extensivas y estudios de las especies de papa en Latinoamérica. Posteriormente en 1940, los científicos ingleses J.G. Hawkes y Balls de Cambrige, luego D.S. Correll de los Estados Unidos y grupos de holandeses, daneses, alemanes y científicos del Centro Internacional de la Papa y Latinoamérica, como el doctor Martín Cárdenas de Bolivia y los profesores Carlos Ochoa y César Vargas de Perú, han contribuido a la realización de numerosas colecciones de material. Todos ellos han desarrollado una labor pionera en la recolección, clasificación y evaluación de las colecciones de papa (Estrada, 1991).

El Centro de Producción y Servicio Toralapa en Cochabamba (FUNDACIÓN PROINPA), actualmente está conservando una colección de especies nativas de Solanum consistente en: cuatro entradas de S. goniocalyx, , una entrada de S. phureja, 148 entradas de $S$. stenotomum, 90 entradas de $S$. $x$ ajanhuiri, 230 entradas de S. x juzepczukii, 65 entradas de S. curtilobum, 939 entradas de S. tuberosum ssp andigena y 63 entradas de $S$. tuberosum ssp tuberosum (PROINPA, 1996). Además el Departamento de Genética y Mejoramiento del PROINPA cuenta con una colección de trabajo con unas 30 especies silvestres para su programa de mejoramiento (Gabriel, et al., 1996b; García, et al., 1996).

\section{Uso de las especies silvestres}

Diversos fitomejoradores han considerado que el uso de las especies silvestres es tediosa, difícil y de larga espera para obtener logros.

Esto posiblemente se ha debido en gran parte a que la mayoría de los fitomejoradores en Europa y Norteamérica han trabajado más que todo en la especie S. tuberosum ssp. tuberosum y sus haploides. En ellos la fertilidad, especialmente del polen, es muy restringida y 
también la viabilidad genética. El problema se vuelve más crítico cuando se híbrida con las especies silvestres (Peloquin et al., 1989, Estrada, 1991; Gabriel, 1994).

Realmente sólo después de 1950, Ross (Estrada, 1991; Gabriel, 1994,), se ha intentado utilizar las especies silvestres, especialmente con los trabajos de holandeses, alemanes, escandinavos, polacos y rusos y algo en Canadá y los Estados Unidos y en Latinoamérica (Wastie, 1991, Rivera-Peña, 1992, Romero, 1993). Sin embargo, estas especies silvestres no pasan de la docena.

La excepción han sido los trabajos de Peloquin (1983) y su equipo de Wisconsin, que utilizaron los haploides de tuberosum con S. phureja o los trabajos de Simmonds (1977) y Glendining (1975) en el Scottish Plant Breeding Station, Escocia y de Plaisted et al. (1975) en Cornell University que lograron obtener tipos neotuberosum, extraídos de la misma $S$. andigena, adaptándola en varias generaciones de selección a los días largos del hemisferio norte, obteniendo así los cultivares neotuberosum.

En Latinoamérica los mejoradotes contaron con ventajas comparativas muy grandes como son la alta fertilidad y la flexibilidad de ploidía que muestran los cultivares nativos (Estrada, 1984a, 1984b, 1990; Estrada y Gabriel, 1991).

La manipulación genética bien dirigida modernamente ha abierto las puertas para explotar mucho más rápidamente, pocas generaciones (dos o tres), los grandes atributos de muchas especies silvestres. Las investigaciones de Mendiburo y Peloquin (1975) y otros han contribuido a desarrollar mejores y más eficientes métodos de mejoramiento (Rivera-Peña, 1992, Niederhauser, 1993).

Estamos generando materiales más acordes a los requerimientos de los agricultores, tratando de obtener variedades del tipo intermedio entre andigena y tuberosum, para lo cual estamos evaluando y utilizando cultivares nativos seleccionados del Banco Nacional de Germoplasma de Tubérculos y Raíces Andinas (BNGTRA) de las especies S. stenotonum como las Qoyllus, S. andigena como las Sakampayas, las Wilas, Palis, las Palas, las Imillas, etc., S. phureja como la Yema de huevo, S. goniocalyx como la Amarilla; y $S$. ajanhuirí como las Lunkus; además de varias especies silvestres como $S$. avilesii y $S$. chacoense como fuentes importantes de resistencia genética para tizón. 


\section{Fitomejoramiento convencional}

\section{Tolerancia a las heladas}

El programa de mejoramiento para tolerancia a heladas fue una continuación del trabajo pionero del Dr. Nelson Estrada (1984a) en Colombia y en el Centro Internacional de la Papa.

Entre las especies silvestres mayormente utilizadas para la obtención de híbridos tolerantes a heladas están: $S$. acaule $(2 n=4 x=48), S$. canasense $(2 n=2 x=24), S$. chomatophilum $(2 n=2 x=24), S$. demissum $(2 n=6 x=72), S$. multidisectum $(2 \mathrm{n}=2 \mathrm{x}=24)$, S. commersonii $(2 \mathrm{n}=2 \mathrm{x}=24)$, S. toralapanum $(2 n=2 x=24)$ y $S$. sanctae rosae $(2 n=2 x=24)$. Su cruzabilidad es variable y pueden tolerar heladas en un rango de -5 a $-8^{\circ} \mathrm{C}$ (PROINPA, 1991,1992, 1993, 1994, 1995, 1996; Estrada et al., 1994a)

Entre las especies cultivadas empleadas para obtener materiales tolerantes a las heladas están: $S$. ajanhuirí $(2 n=2 x=24), S$. andigena $(2 n=2 x=24), S . x$ curtilobum $(2 n=5 x=60)$, S. $x$ juzepczukii $(2 n=3 x=36)$ y $S$. stenotomum $(2 n=2 x=24)$. La cruzabilidad de estas especies varía desde baja a muy buena y toleran temperaturas de -3 a $-5^{\circ} \mathrm{C}$.

\section{Tolerancia a la sequía}

Para obtener tolerancia a la sequía se han utilizado especies silvestres como: S. leptophies, S. medians, S. anamatophylum, S. berthaultii, S. weberbauerii, $S$. mochicense y $S$. megistracrolobum. Estas han sido cruzadas con $S$. phureja y retrocruzadas con cultivares tetraploides de $S$. andigena y $S$. tuberosum.

\section{Resistencia al tizón (Phytophthora infestans)}

Para obtener resistencia durable al tizón ( $P$. infestans), desde 1990 se están utilizando las especies silvestres de solanum (ESS) diploides $(2 n=2 x=24), S$. avilesii, S. bulbocastanum, S. cardiophylum, S. polyadenium y $S$. verrucosum, las ESS tetraploides $(2 n=4 x=48), S$. polytrichon y $S$. stoloniferum, las ESS hexaploides $(2 n=4 x=72 J, S$. iopetalum, $S$. brachycarpum, S. hougasii y S. demissum. La fertilidad de estos materiales es desde muy baja a buena.

Entre las especies cultivadas se han utilizado cultivares de $S$. phureja, $S$. andigena, S. tuberosum, S. stenotomum, S. x juzepczukii y S. goniocalyx, además de otros materiales avanzados provenientes del CIP-Perú e ICAColombia. 


\section{Resistencia a nematodos}

Se han utilizado especies silvestres de S. brevicaule, S. brevidens, S. bulbocastanum, S. gourlayi, S. hondelmanii, S. spegazinii y S. vernei, en cruzamientos con $S$. phureja. Estos materiales mayormente tienen resistencia a Globodera y se han evaluado por su resistencia a Nacobbus aberrans.

Los cultivares de $S$. andigena, que incluyen a los cultivares Gendarme, Ralis, Sakampayas, Jankos, Alka y Sutamari han mostrado buena resistencia principalmente al nematodo-rosario Nacobbus aberrans y han sido cruzadas con clones y cultivares valiosos.

Clones provenientes de la hibridación de $S$. phureja $x$ S. brevidens y $S$. phureja $x$ S. bulbocastanum han mostrado buena resistencia a Nacobbus y han sido cruzados con cultivares tetraploides. Actualmente se están probando híbridos de Phureja-Bulbocastanum, provenientes de fusión asimétrica y simétrica de protoplastos.

El Departamento de Genética y Mejoramiento ha empleado para obtener nuevos cultivares, métodos convencionales, pero modernos, aplicando la manipulación genética de gametos no reducidos $(2 n)$ e híbridos triploides (Figura 1), además de la flexibilidad del abundante germoplasma de especies y cultivares que están siendo conservados en el Centro de Producción y Servicios Toralapa. (Carrasco et al., 1996a; Gabriel et. al., 1996). La Tabla 1 y la Figura 1 muestran que la metodología utilizada para el programa de mejoramiento de papa, permite obtener híbridos con resistencias combinadas a diferentes factores bióticos y abióticos.

Tabla 1. Cruzas y selecciones hechos desde 1990 hasta 1996 y evaluadas por su tolerancia y/o resistencia a tizón, nematodos, heladas y sequía principalmente.

\begin{tabular}{|l|rrrrrrrr|}
\hline \multirow{2}{*}{ Material } & \multicolumn{9}{c}{ CAMPAÑAS AGRÍCOLAS } & \multirow{2}{*}{ Total } \\
\cline { 2 - 8 } & $1990 / 91$ & $1991 / 92$ & $1992 / 93$ & $1993 / 94$ & $1994 / 95$ & $1995 / 96$ & \\
\hline Familias & 117 & 75 & 191 & 255 & 242 & 221 & 1101 \\
\hline Semilla (TS) & 14,430 & 11,000 & 15,000 & 32,000 & 32,882 & 15,000 & 123,312 \\
\hline Plántulas probadas & 12,261 & 7,200 & 9,537 & 8,995 & 23,529 & 9,820 & 71,392 \\
\hline Clones seleccionados & 1,200 & 1,145 & 2,169 & 1,544 & 3,926 & 2,097 & 12,081 \\
\hline \% de selección & 10 & 16 & 23 & 17 & 17 & 21 & 17 \\
\hline
\end{tabular}

Fuente: Gabriel et al. (1996a). 


\section{Logros del fitomejoramiento convencional}

Se presentan resultados de diez años de investigación y mejoramiento en papa realizados en Bolivia, que no excluyen sino complementan, el exitoso trabajo realizado por los anteriores fitomejoradores en Bolivia.

Los resultados que se presentan son novedosos y quizá de poca credibilidad para algunos, porque no han sido logrados por varios investigadores en otras ocasiones y/o épocas en diversas publicaciones. Estos resultados se atribuyen en buena parte a la ventaja comparativa de estar trabajando con las papas en su adecuado ambiente de origen y de contar con la diversidad genética tan vasta de papas nativas y silvestres, principalmente diploides y triploides, que han sido utilizadas como especiespuente para transferir genes valiosos de resistencia de las especies silvestres hacia las cultivadas tetraploides (Estrada, 1991).

La Tabla 1 muestra que hubo un buen uso de los recursos genéticos de papa y que se han generado desde 1990 hasta 1996 aproximadamente 123,312 semillas sexuales pertenecientes a 1,101 familias; de las cuales luego de sembradas y tamizadas, se han obtenido aproximadamente 12,081 clones en fase inicial de selección (Gabriel et al., 1996a).

De pruebas en campo (12,000 clones), se han seleccionado algunos materiales que en la actualidad son clones promisorios y cultivares potenciales que están en validación participativa con agricultores y técnicos y se están multiplicando para obtener semilla de calidad (Gabriel et al., 1996a). 


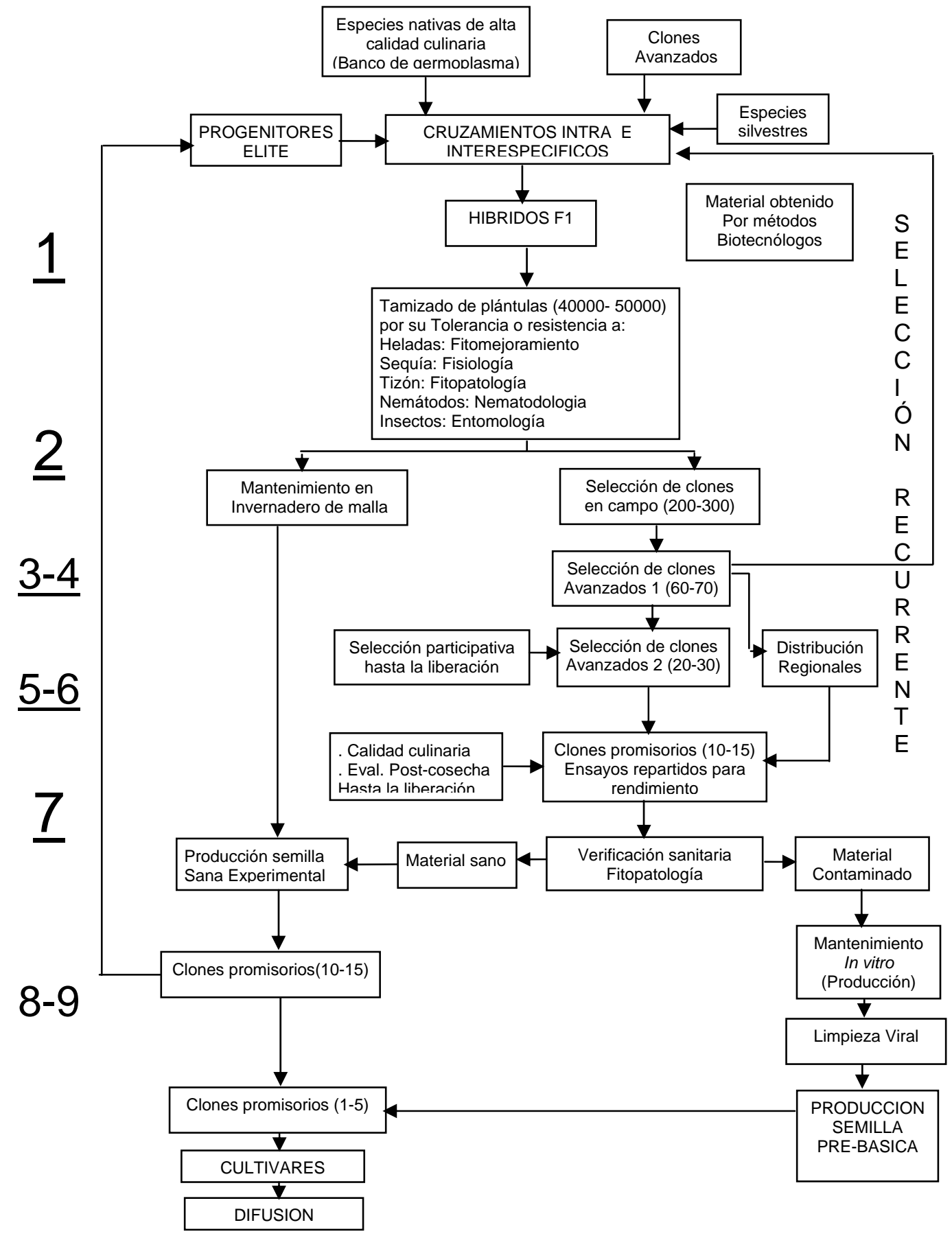

Figura1. Esquema general de mejoramiento genético de papa en Bolivia IBTAPROINPA. 2

2 Carrasco, E., Gabriel, J., García, W. 1996. Dpto. Genética y Mejoramiento. Casilla 4285. IBTA-PROINPA, Cochabamba,Bolivia 


\section{Tolerancia a las heladas y sequía}

Se tienen cruzamientos $\mathrm{F} 1$ de gran valor potencial de: acaule $\mathrm{x}$ phureja $(2 n=3 x=36)$, acaule $x$ chaucha $(2 n=4 x=48)$, megistacrolobum $x$ phureja $(2 n=2 x=24)$, sanctae rosae $x$ phureja $(2 n=2 x-24)$, commersonii $x$ phureja $(2 n=2 x=24)$, stoloniferum $x$ commersonii $(2 n=3 x=36)$, que son resistentes a un promedio de -3 a $-4^{\circ} \mathrm{C}$ de temperatura y tienen alta probabilidad de cruzabilidad con tetraploides.

Se han seleccionado cuatro cultivares tolerantes a heladas, que pueden soportar por dos horas heladas en campo hasta de $-3^{\circ} \mathrm{C} 0-4^{\circ} \mathrm{C}$ por menor tiempo (PROINPA, 1996, García et al., 1996b). Estos cultivares tienen una buena capacidad de recuperación después de la helada, buenos caracteres agronómicos, rendimientos superiores a los cultivares en actual uso en zonas de heladas, hasta en un $100 \%$ o más. Todos son cultivares no amargos (dulces) y su calidad culinaria es aceptable tanto en papa cocida como frita (Tabla 2).

Tabla 2. Nuevos cultivares tolerantes a heladas y sus características más sobresalientes. Campaña 1995-1996.

\begin{tabular}{|c|c|c|c|c|c|}
\hline Cultivar (Clon) & $\begin{array}{l}\text { Color de } \\
\text { tubérculo }\end{array}$ & $\begin{array}{l}\text { Forma } \\
\text { tubérculo }\end{array}$ & $\begin{array}{l}\text { Profundidad } \\
\text { de ojos }\end{array}$ & $\begin{array}{l}\text { Rend } \\
\text { (t/ha) }\end{array}$ & $\begin{array}{l}\text { Calidad } \\
\text { culinaria }\end{array}$ \\
\hline Illimani (90-123-22) & Crema-morado & Redonda & Superficiales & 38 & Buena \\
\hline Totoreña (90-123-18) & Rosado & Redonda & Medianos & 37 & Buena \\
\hline Tunari (90-101-3) & Blanco & Redonda & Medianos & 35 & Aceptable \\
\hline Condori (389349.1) & Morado & Oblonga & Superficiales & 35 & Aceptable \\
\hline Alpha & Blanco & Redonda & Superficiales & 11 & Aceptable \\
\hline Sani Imilla & Morado-crema & Redonda & Profundos & 22 & Buena \\
\hline Luk'y & Blanco & Oblonga & Medianos & 17 & Mala \\
\hline
\end{tabular}

Fuente: PROINPA (1996).

Estos nuevos cultivares son una alternativa ante el serio problema de las heladas en todas las zonas altas de nuestro país. Consideramos que de acuerdo a su adaptación alcanzarán una superficie de por lo menos un 40\% (aproximadamente 5,600 ha) en las zonas con problemas de heladas, teniendo en las mismas una adopción del 100\%. Aunque estas estimaciones son optimistas, nos atrevemos a mencionarlas por las cualidades que hemos observado en estos cultivares a lo largo de los seis años de evaluación.

Respecto de los materiales tolerantes a sequía, estos han sido probados en la Estación Experimental de Chinoli (Potosí). En la actualidad se tiene 
dos cultivares potenciales con buenas características agronómicas, buen rendimiento, bajo porcentaje de marchitez y calidad culinaria aceptable (PROINPA, 1996).

\section{Resistencia al tizón (Phytophthora infestans)}

En 1989 se inició la selección con 135 clones, los cuales provenían del Centro Internacional de la Papa (CIP) y del Instituto Colombiano Agropecuario (ICA), incluyendo como testigos cultivares locales (S. tuberosum ssp. andigena) e introducidos (S. tuberosum ssp. tuberosum), además de cultivares comerciales con resistencia al tizón.

Por selección durante cinco campañas agrícolas se obtuvieron seis nuevos cultivares los cuales mostraron un grado variable de daño por tizón, esto debido principalmente a las condiciones de humedad y temperatura reinantes en las localidades de prueba, lo que determinó la severidad del ataque de las diferentes poblaciones existentes en cada una de ellas.

Las Tablas 3 y 4 muestran los grados de daño alcanzado y los rendimientos de los seis clones que destacaron durante los años de evaluación y se observa que los grados de daño alcanzados por los clones, son siempre menores que los de los testigos, a excepción de los años 1992/93 y 1994/95, en los cuales el testigo resistente mostró menor grado de daño que algunos de los clones, aunque éste no es un indicativo de que su comportamiento haya sido mejor, como se observa en los rendimientos alcanzados.

Los rendimientos obtenidos para los seis clones durante las cinco campañas de evaluación y los testigos Waych'a, Alpha (Susceptibles) y Rosita (Resistente) se observan en la Tabla 4, donde se aprecian los notables rendimientos alcanzados por los clones que son superiores a los testigos e inclusive al testigo resistente. Estos rendimientos expresados en $\mathrm{kg} / \mathrm{planta}$, traducidos a t/ha, considerando una cantidad de 47,000 plantas por hectárea estarían en un rango de 30 a 56 t/ha; rendimientos muy superiores a los obtenidos actualmente, que en el mejor de los casos alcanzan a 25 t/ha con una alta inversión en fungicidas. 
Tabla 3. Área bajo la curva de progreso de la enfermedad (ABCPPI) de seis clones promisorios y tres testigos durante cinco campañas agrícolas.

\begin{tabular}{|l|lllll|}
\hline \multicolumn{1}{|c|}{ Clon } & \multicolumn{5}{|c|}{ Campaña Agrícola } \\
\cline { 2 - 6 } & $1990 / 1991$ & $19991 / 1992$ & $1992 / 1993$ & $1993 / 1994$ & $1994 / 1995$ \\
\hline 575045 & 45.00 & 166.25 & 140.15 & 45.31 & 30.00 \\
720049 & 50.00 & 487.00 & 595.02 & 265.00 & 48.59 \\
676008 & 49.00 & 166.25 & 255.94 & 257.20 & 86.39 \\
385240.2 & 47.00 & 487.50 & 132.40 & 823.80 & 279.84 \\
$84-75-16$ & 31.50 & 68.75 & 265.31 & $1,581.00$ & 157.66 \\
$79-94-3$ & 49.00 & 35.00 & 48.12 & 110.00 & 561.66 \\
Waych'a & 601.25 & $1,803.75$ & 500.00 & $3,294.00$ & $2,601.50$ \\
Alpha & 986.50 & $2,700.00$ & 950.00 & $4,293.00$ & $3,664.00$ \\
Rosita & 542.50 & 750.00 & 458.00 & $1,688.00$ & 208.40 \\
\hline
\end{tabular}

Fuente: Carrasco et al. (1996b).

Tabla 4. Rendimiento en $\mathrm{kg} /$ planta de seis clones promisorios $y$ tres testigos durante cinco campañas agrícolas.

\begin{tabular}{|l|rrrrr|}
\hline \multirow{2}{*}{ Clon } & \multicolumn{5}{|c|}{ Campaña Agrícola } \\
\cline { 2 - 6 } & $1990 / 1991$ & $1991 / 1992$ & $1992 / 1993^{*}$ & $1993 / 1994^{*}$ & $1994 / 1995^{*}$ \\
\hline 575045 & 0.93 & 0.90 & 1.19 & 1.20 & 1.16 \\
720049 & 0.91 & 0.82 & 1.13 & 0.52 & 0.80 \\
676008 & 1.10 & 0.65 & 1.09 & 0.80 & 0.82 \\
385240.2 & 0.95 & 0.88 & 1.07 & 0.72 & 0.85 \\
$84-75-16$ & 1.18 & 0.69 & 0.81 & 0.63 & 0.81 \\
$79-94-3$ & 0.76 & 0.99 & 1.00 & 1.03 & 0.69 \\
Waych'a & 0.46 & 0.15 & 0.54 & 0.07 & 0.23 \\
Alpha & 0.52 & 0.19 & 0.54 & 0.06 & 0.18 \\
Rosita & 0.65 & 0.47 & 0.77 & 0.11 & 0.52 \\
\hline
\end{tabular}

* Promedio de cuatro repeticiones.

Fuente: Carrasco et al. (1996b).

En la campaña 1994/95 fueron preliberados en Cochabamba, seis nuevos cultivares cuyas características agronómicas podemos observar en la Tabla 6. De estos, en la actualidad, tres cultivares como Robusta, Jaspe e India han sido priorizados por los agricultores y están en plena etapa de difusión. En Chuquisaca los cultivares preliberados Chotas Ñawi y Cordillera también están en plena etapa de difusión con agricultores. 


\section{Resistencia a nematodos}

Después de cuatro años de evaluación y selección en el campo se han obtenido nueve clones promisorios que han sido caracterizados agronómicamente en los tubérculos (forma, uniformidad, profundidad de ojos, color de piel y color de carne) y evaluados por su rendimiento. También se hicieron pruebas de calidad culinaria y de bromatología (García et al., 1996a).

La selección del material genético por resistencia a nematodos, más las caracterizaciones agronómicas, han permitido obtener tres de los mejores clones que destacaron en rendimiento y resistencia y/o tolerancia a nematodos a través de cuatro campañas agrícolas. Con estos cultivares (Jaspe, Gendarme y Puka uya) se obtendrán rendimientos con 18\% a 35\% más que el cultivar local Waych'a. Estos clones en la actualidad se encuentran en fase de limpieza viral y multiplicación para su posterior difusión.

\section{Selección participativa de variedades (SPV)}

\section{SPV 1990-1994}

El primer año de selección participativa los agricultores evaluaron 135 clones de los fitomejoradores. No se utilizó material genético local porque aún no se había generado. Los clones evaluados fueron del CIP-Perú e ICA-Colombia (Bejarano et al., 1992).

Los clones seleccionados por los agricultores el primer año fueron almacenados y sembrados con otros clones seleccionados por los fitomejoradores el siguiente año. Durante 1993-94 se seleccionaron 10 clones promisorios de 133 evaluados (Tabla 6). La importancia de los agricultores en el manejo de los ensayos fue incrementando progresivamente. Las técnicas de evaluación con agricultores cambiaron con el incremento del conocimiento de los criterios de selección de los agricultores. Nuestro conocimiento de cómo involucrar a los agricultores mejoró.

Esta SPV fue realizada por los científicos sociales junto con ensayos convencionales realizados por los fitomejoradores. De estos trabajos resultó la liberación de seis nuevas variedades de papa resistentes al tizón (Carrasco et al., 1997) (Tabla 5). 
Tabla 5. Principales características de los clones con resistencia al tizón de la papa ( $P$. infestans liberados en Cochabamba, Bolivia. (1994-1995).

\begin{tabular}{|c|c|c|c|c|}
\hline Clon & Nombre & Genealogía & $\begin{array}{c}\text { Características } \\
\text { principales }\end{array}$ & $\begin{array}{l}\text { Reacción a otros } \\
\text { factores }\end{array}$ \\
\hline 575045 & Perla & $\begin{array}{c}\text { POOS-16 } \\
\text { (tbr Holanda) }\end{array}$ & $\begin{array}{l}\text { Tub. redondeados, } \\
\text { ojos superf., piel } \\
\text { crema, carne crema }\end{array}$ & $\begin{array}{l}\text { Manchas Foliares } \\
\text { (MF) -Tolerante } \\
\text { Heladas-Toler. }\end{array}$ \\
\hline 720049 & Puquina & $\begin{array}{l}\text { Furore* } \\
\text { (US 129.2 } \\
\text { Kathadin) }\end{array}$ & $\begin{array}{l}\text { Tub. redondeados, } \\
\text { ojos superf., piel } \\
\text { rosada, carne crema }\end{array}$ & MF-Tolerante \\
\hline 676008 & India & $\begin{array}{c}\text { US 136.6 } \\
*(3345 \mathrm{D}(1) \\
* 2288 \mathrm{~A}(2))\end{array}$ & $\begin{array}{l}\text { Tub. redondeados, } \\
\text { ojos medianos, piel } \\
\text { rosada, carne crema }\end{array}$ & $\begin{array}{l}\text { MF-Tolerante } \\
\text { Verruga- } \\
\text { Moderadamente } \\
\text { resistente }\end{array}$ \\
\hline 385240.2 & Chaposa & $\begin{array}{l}\text { AVRDC-1287.19* } \\
\text { (BL-2)9 }\end{array}$ & $\begin{array}{l}\text { Tub. redondeados, } \\
\text { ojos medianos, carne } \\
\text { crema halo rosado, } \\
\text { carne crema }\end{array}$ & MF-Tolerante \\
\hline $84-75-16$ & Jaspe & $\begin{array}{l}(\text { sto*bre) })^{*} \\
\text { (tbr*adg) }^{*}\end{array}$ & $\begin{array}{c}\text { Tub. redondeados, } \\
\text { ojos mediano, piel } \\
\text { crema halo morado, } \\
\text { carne crema }\end{array}$ & $\begin{array}{l}\text { Nacobbus-Resistente } \\
\text { Sequía-Tolerante }\end{array}$ \\
\hline $79-94-3$ & Robusta & $($ tbr*adg)*tbr & $\begin{array}{l}\text { Tub. comprimido, ojos } \\
\text { medianos, piel rosado- } \\
\text { crema, carne crema }\end{array}$ & $\begin{array}{c}\text { MF-Tolerante } \\
\text { Verruga- Resistente }\end{array}$ \\
\hline
\end{tabular}

Fuente: Carrasco et al. (1996b) y Quiroga (1996).

Logros y dificultades. Varios métodos para evaluación de clones fueron probados y se aprendió mucho acerca de los criterios de los agricultores en la selección de nuevas variedades. Los fitomejoradores asimilaron los resultados de la evaluación con agricultores y prestaron más atención al color, forma y tamaño de los tubérculos al seleccionar germoplasma mejorado.

En 1990-91, la comunicación entre científicos sociales y fitomejoradores fue menos que ideal y no todos los fitomejoradores estaban de acuerdo en involucrar al agricultor cuando hay un gran número de clones.

\section{SPV 1995-1999}

En este periodo la comunicación entre los científicos sociales y fitomejoradores mejoró respecto del anterior periodo y se observó que 
trabajaron conjuntamente. Sobre la base de sus experiencias en 
los años anteriores, desarrollaron metodologías para involucrar a los agricultores en las diferentes fases de selección del material, para lograr resultados de evaluación que fueran comparables en sitios y en el tiempo. Los científicos sociales todavía siguieron realizando las SPV, en el entendido de que los fitomejoradores tomarían la responsabilidad progresivamente.

Logros y dificultades. La metodología para involucrar a los agricultores en la evaluación de clones es utilizada para todos los trabajos de fitomejoramiento y la mayoría de los científicos en PROINPA aceptan que la participación de los agricultores necesita ser permanente e institucionalizada.

En 1999 la experiencia de evaluación de clones promisorios con agricultores en la floración (Gabriel et al., 1999) mostró que los agricultores de Compañía Pampa y Piusilla en Morochata (Cochabamba) destacaron el vigor, la sanidad de las plantas y la resistencia como los factores más importantes. En orden de preferencia eligieron en primer lugar al clon 85-87-3 en Compañía Pampa y el clon 82-3-5 X79-94-3 en Piusilla (Tablas 6 y 7 ).

Tabla 6. Evaluación y selección participativa con agricultores de la comunidad de Compañía Pampa. Campaña agrícola 1998-99.

\begin{tabular}{|c|c|c|c|c|c|c|}
\hline \multirow{2}{*}{ Cultivar } & \multicolumn{2}{|c|}{$\begin{array}{c}\text { Floración } \\
\text { (6 agricultores) }\end{array}$} & \multicolumn{2}{|r|}{$\begin{array}{c}\text { Cosecha } \\
\text { (6 agricultores) }\end{array}$} & \multicolumn{2}{|c|}{$\begin{array}{c}\text { Calidad culinaria } \\
\text { (8 agricultores) }\end{array}$} \\
\hline & Orden & Razones & Orden & Razones & Calificación & Razones \\
\hline $85-87-3$ & 1ro. & $\begin{array}{l}\text { Buen tamaño } \\
\text { de la planta } \\
\text { resistente al } \\
\text { tizón, tallos } \\
\text { gruesos }\end{array}$ & 2do & $\begin{array}{l}\text { Tiene mucho } \\
\text { tubérculo pequeño, } \\
\text { le falta cara, ojos un } \\
\text { poco profundos }\end{array}$ & Buena & $\begin{array}{l}\text { Harinosa, } \\
\text { color blanco } \\
\text { de la carne }\end{array}$ \\
\hline 387179.1 & 2 do. & $\begin{array}{l}\text { Susceptible } \\
\text { amarillamiento } \\
\text { (Alternaria) }\end{array}$ & 3ro & $\begin{array}{l}\text { Color de la carne } \\
\text { amarilla }\end{array}$ & Regular & $\begin{array}{l}\text { Medio } \\
\text { aguanosa }\end{array}$ \\
\hline $\begin{array}{c}82-3- \\
5 \times 79-94-3\end{array}$ & 3ro. & $\begin{array}{l}\text { Tiene ataque } \\
\text { de tizón un } \\
\text { poco }\end{array}$ & 1ro & $\begin{array}{l}\text { Buen rendimiento, } \\
\text { tubérculos grandes, } \\
\text { parece R. Toralapa }\end{array}$ & Buena & $\begin{array}{l}\text { Harinosa, } \\
\text { color blanco } \\
\text { de la carne, } \\
\text { parece } \\
\text { Waych'a }\end{array}$ \\
\hline $\begin{array}{c}\mathrm{R} . \\
\text { Toralapa }\end{array}$ & 4to. & $\begin{array}{l}\text { Es menos } \\
\text { resistente al } \\
\text { tizón que el } \\
\text { tercero }\end{array}$ & 4to & $\begin{array}{l}\text { poco rendimiento } \\
\text { tubérculos muy } \\
\text { pequeños }\end{array}$ & Regular & $\begin{array}{l}\text { Medio } \\
\text { aguanosa }\end{array}$ \\
\hline
\end{tabular}

Fuente: Gabriel et al. (1999). 


\section{Tabla 7. Evaluación y selección participativa con agricultores de la comunidad de Piusilla. Campaña agrícola 1998-99.}

\begin{tabular}{|c|c|c|c|c|c|c|}
\hline \multirow{2}{*}{ Cultivar } & \multicolumn{2}{|c|}{$\begin{array}{c}\text { Floración } \\
\text { (6 agricultores) } \\
\end{array}$} & \multicolumn{2}{|c|}{$\begin{array}{c}\text { Cosecha } \\
\text { (6 agricultores) } \\
\end{array}$} & \multicolumn{2}{|c|}{$\begin{array}{l}\text { Calidad culinaria } \\
\text { (8 agricultores) }\end{array}$} \\
\hline & Orden & Razón(es) & Orden & Razón(es) & Calificación & Razón(es) \\
\hline $85-87-3$ & 2do. & $\begin{array}{l}\text { Tallos } \\
\text { delgados }\end{array}$ & 3ro. & $\begin{array}{l}\text { Tiene forma de } \\
\text { papa runa }\end{array}$ & Regular & $\begin{array}{l}\text { Poco } \\
\text { aguanosa }\end{array}$ \\
\hline 387179.1 & 3ro. & $\begin{array}{l}\text { Follaje muy } \\
\text { grande }\end{array}$ & 2do & $\begin{array}{l}\text { Color de la } \\
\text { carne } \\
\text { amarilla }\end{array}$ & Regular & $\begin{array}{l}\text { Poco } \\
\text { Aguanosa, } \\
\text { color de carne } \\
\text { amarillo, no le } \\
\text { gusta al } \\
\text { consumidor }\end{array}$ \\
\hline $82-3-5 \times 79-94-3$ & 1 ro. & $\begin{array}{l}\text { Resistente, } \\
\text { tallos gruesos, } \\
\text { buen tamaño } \\
\text { de } \\
\text { follaje }(50 \mathrm{~cm})\end{array}$ & 1 ro. & $\begin{array}{l}\text { Buen } \\
\text { rendimiento } \\
\text { parecida en } \\
\text { forma a la } \\
\text { Waych'a }\end{array}$ & Buena & $\begin{array}{l}\text { Harinosa, } \\
\text { parece } \\
\text { Waych'a }\end{array}$ \\
\hline Waych'a & 4to & $\begin{array}{l}\text { No resiste el } \\
\text { tizón }\end{array}$ & 4to. & $\begin{array}{l}\text { Bajo } \\
\text { rendimiento }\end{array}$ & Buena & $\begin{array}{l}\text { Harinosa. } \\
\text { Buena carne } \\
\text { de color blanco }\end{array}$ \\
\hline
\end{tabular}

Fuente: Gabriel et al. (1999).

En la cosecha en ambas comunidades los criterios de selección fueron el rendimiento, el tamaño del tubérculo, la semejanza con otras variedades conocidas, la apariencia y color de la piel y la profundidad de los ojos. El clon 82-3-5x79-94-3 mostró ser de primera preferencia para los agricultores.

La evaluación culinaria realizada al mes de la cosecha, mostró que en la comunidad de Piusilla la variedad local Waych'a es la más preferida, siguiendo en importancia el clon 82-3-5x79-94-3, por su similitud a la variedad Waych'a.

Seguimos analizando cuándo la participación del agricultor debe empezar y cómo deben integrarse las evaluaciones del agricultor y del investigador. Aunque los fitomejoradores y los científicos sociales han aprendido a trabajar estrechamente, hasta 1998 todavía no se había podido reflejar propiamente en los informes de investigación, en parte debido a un formato institucional que tendía a separar los resultados de la investigación a través de disciplinas. Sin embargo ahora este aspecto está tratando de ser superado, debido a que tenemos un nuevo formato institucional (proyectos). 


\section{Síntesis de los logros de la SPV}

Se formaron grupos de evaluadores de 10 personas incluyendo a hombres y mujeres quienes evalúan separadamente los materiales. En sociedad con el proyecto IPRA (Investigación Participativa en Agricultura) del CIAT, Colombia, PROINPA se especializó en un centro para el entrenamiento en los métodos participativos y se establecieron los comités locales de investigación agrícola (CIAL). Como resultado de los métodos promovidos por el IPRA, estos se han convertido en una práctica normal para los científicos del PROINPA. El método de orden de preferencia es la técnica principal que se ha utilizado para evaluar 10 a 30 clones por sus características morfológicas (Ashby 1992). Los fitomejoradores son responsables principalmente del manejo de estos materiales los cuales son sembrados en parcelas de agricultores para ser evaluados. Los clones con puntaje más alto por rendimiento y resistencias son evaluados para color del tubérculo, forma de tubérculo y por sus propiedades culinarias usando la evaluación absoluta. Los clones seleccionados son proporcionados a los agricultores para que sean probados por ellos mismos.

Los agricultores involucrados en SPV en los sitios de altura están multiplicando las variedades más promisorias para su uso como semilla por agricultores de zonas más bajas que están interesados en cultivar variedades resistentes. La producción de tubérculo-semilla tiene una tasa de multiplicación más baja que los cereales. La SPV y el uso de semilla a través del sistema informal están ayudando a superar el cuello de botella de la multiplicación (Thiele, 1999).

\section{Mejoramiento participativo (MP): 1997-1998}

Los fitomejoradores en PROINPA han iniciado una experiencia piloto de Mejoramiento Participativo. Los científicos sociales jugaron un papel de apoyo. Los Fitomejoradores de PROINPA se animaron al MP por el éxito de la familia de agricultores fitomejoradores que liberaron nuevos cultivares de papa en Ecuador (Bastidas, 1991).

Esta experiencia se inició con la intención de ver si el MP funciona y desarrollar metodologías de capacitación eficiente para que los agricultores pudieran lograr cultivares mejorados con alto rendimiento potencial y resistencia, utilizando recursos genéticos locales, adaptados a nichos específicos. La localidad de Morochata fue escogida como el sitio de prueba debido a la existencia de buenas relaciones con los agricultores que han venido trabajando con SPV y la presencia del CIAL (Organización de agricultores que hacen investigación), quienes estaban interesados en participar. 
Para iniciar un programa de mejoramiento genético con los agricultores, lo primero que se realizó fue implementar una guía de capacitación preliminar donde se trató de explicar de la manera más sencilla posible sobre algunos conceptos fundamentales de genética como es la variabilidad genética y de su manipulación para el mejoramiento genético. Principalmente se utilizaron elementos comprensibles para el agricultor, como la familia del agricultor que está constituida de padres e hijos. Se explicó que los hijos (en este caso las plantas de papa), aún cuando son hermanos completos no son iguales entre sí, sino que cada uno tiene un fenotipo y genotipo diferente, lo cual hace que se tengan diferentes reacciones a los factores abióticos y bióticos que causan enfermedades. Luego que se comprendieron estos principios básicos se enseñó a los agricultores que al igual que en los humanos y los animales, en las plantas también pueden diferenciarse órganos femeninos y masculinos. Esto se hizo llevando a los agricultores algunas inflorescencias de papa. Los agricultores fueron capaces de diferenciar e identificar las anteras (órgano masculino) y el estigma (órgano femenino) en las flores.

Así, los agricultores aprendieron a escoger las plantas a ser utilizadas como el progenitor macho (anteras de planta seleccionada) y al hembra (poseedor del estigma).

Previamente a la combinación genética de los cultivares, lo agricultores identificaron sus principales problemas, señalando al tizón (Phytophthora infestans) y al nematodo-rosario (Nacobbus aberrans) como los más importantes. También identificaron con mucha propiedad, qué cultivares existentes en sus zonas podrían ser utilizados en un programa de mejoramiento genético. Identificaron tres cultivares, Waych'a, que es un cultivar de la especie andigena y posee genes menores de resistencia al tizón y buena calidad culinaria, Gendarme que también es una andigena con buena calidad culinaria y resistencia al nematodo - rosario, cultivar seleccionado del BNGTRA en 1993 - 1995 y Runa Toralapa (Perricholi), que es un híbrido con una alta resistencia al tizón.

Para hacer los cruzamientos se capacitó a cinco agricultores del Comité de Investigación Agrícola Local (CIAL), quienes mostraron interés en participar en esta nueva experiencia. Se decapitaron inflorescencias de los cultivares mencionados y se escogió a Waych'a como macho por la buena fertilidad de polen que posee, para combinarla con la Gendarme y la Runa Toralapa. Se han utilizando materiales fáciles de disponer como una tapa de pasta dental para recolectar el polen y etiquetas de cartulina elaboradas por los agricultores, para identificar las cruzas realizadas. Fueron los mismos agricultores quienes realizaron los cruzamientos. Al mes se cosecharon las bayas y se obtuvo la semilla sexual, que fue sembrada en almacigos caseros. 
Logros y dificultades. Los agricultores están fascinados en hacer los cruzamientos y muy interesados en continuar con esta experiencia. Hemos empezado a desarrollar materiales de capacitación basados en charlas y prácticas en el campo que serán compartidas en sesiones de educación no formal.

\section{Perspectivas futuras}

Es necesario que se emplee para el mejoramiento de la papa tecnología moderna de manipulación de ploidía y biotecnología, utilizando haploides, gametos $2 n$, cultivo de células, callos, fusiones asimétricas con sólo fracciones de cromosomas, fusiones mitóticas de células, plantas transgénicas, etc. Estas tecnologías modernas permitirán la identificación de la base molecular para la tolerancia a las heladas y demás enfermedades y transferir la misma de $\mathrm{S}$. acaule. S. commersonii, S. chomatophilum y S. brevidens a papas cultivadas (fusión asimétrica de protoplastos).

Hemos aprendido mucho acerca de la preferencia de los agricultores, sin embargo, no hemos sistematizado toda la información. Pretendemos realizar un mapeo o modelo del ideotipo de papa que el agricultor desearía cultivar.

Esperamos involucrar a ONGs e instituciones de desarrollo en este trabajo. El plan es desarrollar una guía de capacitación que se pueda utilizar para la capacitación de agricultores en MP. Nuestra hipótesis es que con mayor participación de agricultores e instituciones podrán ser generados nuevos materiales genéticos para problemas específicos en casos donde el PROINPA no trabaja, a un costo probablemente más bajo.

Además, en esta forma el MP podrá contribuir al mantenimiento de la diversidad genética en el campo del agricultor. Los materiales mejorados serán, por su rendimiento y resistencias, menos vulnerables al reemplazo o abandono. Usando los cultivares nativos como parentales y seleccionando en sus progenies se logrará mantener gran parte de los genes iniciales (teóricamente). La selección de las progenies por el agricultor podrá resultar en un cultivar mejorado que no es genéticamente homogéneo, lo cual podrá tener ventajas en relación de resistencias y mantenimiento de diversidad in situ. 
Sin embargo, hasta ahora esta es una experiencia piloto. PROINPA continuará haciendo fitomejoramiento convencional complementada por SPV, para la resistencia durable al tizón y otros factores. Esperamos documentar nuestras experiencias en MP y comparar los costos y beneficios respecto del mejoramiento convencional. Si la experiencia en MP muestra que es ventajosa, por lo menos bajo algunas circunstancias, entonces empezaremos a promoverla más ampliamente. Aún cuando el MP no demuestre ser ventajoso nos ayudará a aprender más sobre las decisiones del agricultor en la selección de cultivares.

\section{Agradecimientos}

Los autores agradecen la colaboración de todos los colegas de la Fundación PROINPA, al CIP-Perú, ARADO, SEPA, PROSEMPA, ASAR que se han involucrado en la investigación y la generación de nuevas variedades y al Dr. Enrique Fernández-Northcote por sus valiosas contribuciones en el Manejo Integrado del Tizón en Bolivia.

\section{Referencias Bibliográficas}

1. Ashby, J.A. 1990. Evaluating technology with farmers: a handbook. Cali, CIAT.

2. Bastidas, G. 1991. Agricultores genetistas. Documento preparado para la XV Reunión de la Asociación Latinoamericana de la Papa, 8 a 14 septiembre 1991, Lima.

3. Bejarano, C.; Watson, G.; Estrada, N.; Gabriel, J. 1992. Evaluación y selección de factores por agricultores y técnicos en clones de papa con resistencia al tizón tardío (Phytophthora infestans) Revista de Agricultura. Co-edición. F.C.A.P.-UMSS, IBTA-PROINPA. Cochabamba, septiembre, p. 34-41. 
4. Carrasco, N.; Gabriel, J.; García, W. 1996a. Estrategias y perspectivas del mejoramiento genético de la papa en Bolivia. En: Documento Marco. Cochabamba, Bolivia. $25 \mathrm{p}$.

5. Carrasco, E.; Estrada, N.; Gabriel, J.; Alfaro, G.; Larondelle, Y.; García, W.; Quiroga, O. 1997. Seis cultivares potenciales de papa con resistencia al tizón tardío (Phytophthora infestans) en Bolivia. Revista Latinoamericana de la Papa 9/10:106-122.

6. Carrasco, E.; Estrada, N.; Gabriel, O.; García, W.; Mendoza, O.: Quiroga, O. 1995. Seis nuevos cultivares de papa con resistencia al tizón. TecnoIBTA 1(5):1-8.

7. De Franco, M.; Godoy, R. 1993. Potato-Led Growth: The macroeconomic effects of technological innovation in Bolivian agriculture. The Journal of Development Studies 29(3):561-587.

8. Estrada, N. 1884a. Acaphu: a tetraploid, fertile breeding line, selected from S. acaule x S. phureja cross. Am. Pot. J. 61:1-8.

9. Estrada, N. 1884b. Taxonomía y mejoramiento de la papa. En: Manual sobre manejo de germoplasma de papa. Documento de capacitación. Centro Internacional de la Papa, Lima, Perú. p. 43-48

10. Estrada, N. 1991. Especies silvestres de la papa y los cultivares nativos de importancia en el mejoramiento genético de la papa. En: Conferencia de la XIV reunión de la ALAP, Lima, Perú. 26 p.

11. Estrada, N. 1990. Híbridos triploides derivados de Solanum stoloniferum Schlecht et Beché y su valor en el mejoramiento de la papa. Revista Latinoamericana de la Papa 3:80-87.

12. Estrada, N.; Carrasco, E.; García, W. 1994a. Utilización de varias especies silvestres y cultivadas para el mejoramiento genético de la papa. En: Carol Perpich (Ed.). Primera Reunión de Recursos Genéticos de la Papa, Raíces y Tubérculos Andinos. Cochabamba, Bolivia. p. 65-75

13. Estrada, N.; Gabriel, J. 1991. Utilización de Solanum bulbocastanum en el mejoramiento de la papa. XIV Reunión ALAP, Sep. 8-14, Lima, Perú.

14. Estrada, N.; Fernández-Northcote, E.; Carrasco, E.; Navia, O. 1994b. Mejoramiento genético para resistencia enfermedades y plagas de la papa en Bolivia. En: Memorias del $1^{\text {er }}$ Taller sobre Resistencia Duradera en Cultivos Alto Andinos de Bolivia, Colombia, Ecuador y Perú. Quito, Ecuador. $111 \mathrm{p}$. 
15. Gabriel, J. 1994. Resistencia de híbridos interespecíficos de papa al tizón tardío (Phythopthora infestans (Mont.) de Bary) y su caracterización citológica. Tesis de maestría. Colegio de Postgraduados, Montecillo, Edo. México, México. 94 p.

16. Gabriel, J.; Carrasco, E.; García, W., Mendoza, O., Torrez, R., Bustamante, J. 1996. Resistencia Al Tizón (Phytophthora infestans (Mont.) De Bary) en Clones Promisorios De Papa. En: Compendio de la IV reunión de la Papa. 8-11 octubre, 1996. Cochabamba, Bolivia. p. 147

17. Gabriel, J., Carrasco, E.; García, W.; Mendoza, O. 1996b. Uso de los Recursos Fitogenéticos de Papa en el Mejoramiento Genético. En: Compendio de la IV reunión de la Papa. 8-11 octubre, 1996. Cochabamba, Bolivia. p. 149.

18. Gabriel, J.; Torrez, R.; Plata, G. 1999. Investigación participativa en la evaluación y selección de clones promisorios resistentes al tizón de la papa (Phytophthora infestans) en Cochabamba. En: Daniel L. Danial (Eds.). Tercer Taller de PREDUZA en Resistencia Duradera en cultivos Altoandinos. 27-29 sept., Cochabamba, Bolivia. p. 176-187.

19. García, W.; Oros, R.; Gabriel, J.; Carrasco, E.; Mendoza, O.; Franco, J.; Diaz, O. 1996. Tres nuevos cultivares de papa con resistencia y/o tolerancia a Nacobbus aberrans y Globodera spp. En: Memorias de la IV Reunión de la Papa. 8-11 octubre, 1996, Cochabamba, Bolivia. p. 159, 160.

20. García W.; Carrasco E.; Gabriel J.; Mendoza O.; Luján J. C. 1996. Nuevos cultivares de papa tolerantes a heladas en Bolivia. En: Memorias de la IV Reunión de la Papa. 8-11 octubre, 1996, Cochabamba, Bolivia. p. 157 158.

21. Glendining, D.R. 1975. Neotuberosum: new potato breeding material. Potato Res. 18:256-261; 343-350; 50:351-362.

22. Hawkes, J. G. 1988. The evolution of cultivated potatoes and their tuberbearing wild relatives. Kurturpflnze 36:189-208.

23. Hooker, W.J. 1981. Compendio de enfermedades de la papa. Centro Internacional de la Papa, Lima, Perú. 165 p.

24. Mendiburo, A. O.; Peloquin, S.J. 1976. Sexual poly-ploidization: some terminology and definitions. Theor. Appl. Gen. 48:142-144.

25. Niederhauser, J. 1993. International cooperation in potato research and development. Ann. Rev. Phytopathol. 31:1-21.

26. Peloquin, S.J. 1983. New approaches to breeding for the potato for year 2000. Research for the potato in year 2000. CIP. p. 32-34. 
27. Peloquin, S.J.; Jansky, S.H.; Yerk, G.L. 1989. Potato cytogenetics and germplasm utilization. Am. Potato J. 66:629-638.

28. Plaisted, R.L.; Thurston, H.D.; Tingey, W.M. 1975. Five cycle of selection within a population of Solanum tuberosum ssp andigena. Am. Potato J. 52:280 (Abstr.)

29. Programa de Investigación de la Papa (PROINPA). 1991. Informe Anual PROINPA, 1990-1991. Cochabamba, Solivia. 260 p.

30. Programa de Investigación de la Papa (PROINPA). 1992. Informe Anual PROINPA, 1991-1992. Cochabamba, Bolivia. 297 p.

31. Programa de Investigación de la Papa (PROINPA). 1993. Informe Anual PROINPA, 1992-1993. Cochabamba, Bolivia.

32. Programa de Investigación de la Papa (PROINPA). 1994. Informe Anual PROINPA, 1993-1994. Cochabamba, Bolivia. 620 p.

33. Programa de Investigación de la Papa (PROINPA). 1995. Informe Anual PROINPA, 1994-1995. Cochabamba, Bolivia. 900 p.

34. Programa de Investigación de la Papa (PROINPA). 1996. Informe Anual PROINPA, 1995-1996. Cochabamba, Bolivia. 201 p.

35. Quiroga, O. 1996. Caracterización y evaluación de clones promisorios de papa resistentes al tizón (Phytophthora infestans (Mont.) de Bary). Tesis Ing. Agr. Universidad Técnica de Oruro, Facultad de Agronomía, Oruro, Bolivia. $100 \mathrm{p}$.

36. Rivera-Peña, A. 1992. Use of wild tuber-bearing species of Solanum for breeding potatoes against Phythopthora infestans (Mont.) de Bary. En: Rousselle-Bougeois and Rousselle (eds.). Proceeding of Joint Conference of the EAPR Breeding and Varietal Assessement Section and the EUCARPIA Potato Section. Landernau, France, 12-17 January. p. 19-24.

37. Romero, C.S. 1993. Hongos fitopatógenos. Universidad Autónoma de Chapingo, México. 347 p.

38. Simmonds, N. W. 1977. Neotuberosum and the genetic base in potato breeding, ARC Research Rev. Scott PI Breed Sta. 2:9-11.

39. Thiele, G. 1999. Informal potato seed systems in the Andes: why are they important and what should we do with them? World Development, 27(1):83-100.

40. Thiele, G.; Gardner, G.; Torrez, R.; Gabriel, J. 1997. Farmer involvement in selecting new varieties: potatoes in Bolivia. Experimental Agriculture 33:1-16.

41. Wastie, R.L. 1991. Breeding for resistance. En: D.D. Ingram and P.H. Williams (eds.). Phytophthora infestans, the cause of late blight of potato. Vol. 7. Academic Press, London. p. 192-224. 